\title{
Inferior Phrenic Artery, Variations in Origin and Clinical Implications - A Case Study
}

\author{
${ }^{1}$ Dr.Anupama D, Dr.R.Lakshmi Prabha Subhash ${ }^{2}$.Dr. B.S Suresh ${ }^{3}$ \\ Assistant Professor. Dept. Of Anatomy, SSMC. Tumkur.Karnataka.India \\ Professor \& HOD. Dept. Of Anatomy, SSMC. Tumkur.Karnataka.India \\ Associate professor.Dept. Of Anatomy, SSMC. Tumkur.Karnataka.India
}

\begin{abstract}
Variations in the branching pattern of abdominal aorta are quite common, knowledge of which is required to avoid complications during surgical interventions involving the posterior abdominal wall. Inferior Phrenic Arteries, the lateral aortic branches usually arise from Abdominal Aorta, just above the level of celiac trunk. Occasionally they arise from a common aortic origin with celiac trunk, or from the celiac trunk itself or from the renal artery. This study describes the anomalous origin of this lateral or para aortic branches in the light of embryological and surgical basis. Knowledge of such variations has important clinical significance in abdominal operations like renal transplantation, laparoscopic surgery, and radiological procedures in the upper abdomen or invasive arterial procedures .
\end{abstract}

Keywords: Abdominal Aorta, Celiac Trunk(Ct), Diaphragm, Inferior Phrenic Artery (Ipa), Retro Peritoneal, Renal Artery $(R a)$.

\section{Introduction}

The abdominal aorta begins from the level of $12^{\text {th }}$ thoracic vertebra after passing through the Osseo aponeurotic hiatus of diaphragm. It courses downwards with Inferior vena cava to its right and terminates at the level of $4^{\text {th }}$ lumbar vertebra by dividing in to two terminal branches. The branches of abdominal aorta can be classified as splanchnic (visceral) and parietal branches. Inferior phrenic arteries (aa. phrenica inferiores) are two small lateral aortic branches, which supply the diaphragm, but present much variety in their origin and supplementary phrenic vessels are common.1, 2. They may arise separately from the lateral aspect of aorta, immediately above the celiac trunk, or by a common trunk, which may spring either from the aorta or from the celiac trunk. Sometimes one is derived from aorta and other from celiac trunk. Other sources may be renal, supra renal, hepatic ,left gastric or superior mesenteric arteries in $8 \%$ of the cases. $2,3,4$. They diverge from one another across the crura of the diaphragm, and then run obliquely upward and laterally along its its inferior surface.

The left phrenic artery passes behind the esophagus, and runs forward on the left side of the esophageal hiatus. The right phrenic artery passes behind the inferior vena cava, and along the right side of the caval opening. Posterior to the central tendon, each vessel divides into a medial and a lateral branch. The medial branch curves forward, and anastomoses with its fellow of the opposite side, and with the musculo phrenic and pericardio phrenic arteries. The lateral branch passes towards the thorasic wall, and anastomoses with the lower intercostal arteries, and with the musculo phrenic artery. The lateral branch of the right phrenic artery gives off few branches to the inferior vena cava; and the left one, to esophagus. Each vessel gives off superior suprarenal branches to the suprarenal gland of its own side. The capsule of liver and spleen also receive a few twigs from the right and left arteries respectively.1,2. Studies regarding the IPA are limited since they were considered to be of minor clinical interest but nowadays it is known that they form an important source of extra hepatic arterial source of blood supply in Hepato cellular carcinoma.5 As it may also arise in common with renal and supra renal arteries, due care should be taken to avoid ligating it during the surgeries involving Kidney and adrenal glands 3 . Hence present study is done to analyze the variations in the mode of origin of IPA and its clinical implications thereof.

\section{Methodology}

Retro peritoneal region of 50 embalmed cadavers were thoroughly cleaned, carefully dissected and studied in detail for the origin of right and left IPAs in the Dept of Anatomy, SSMC, Tumkur. Dissection was done according to the guidelines given in Cunningham's Mannual . After resection of tissues subjacent to the diaphragmatic crura and tissues along the median arcuate ligament, structures were exposed, allowing for observation of the origin of the IPA. Photographs of the same were taken.

\section{Observations}


In the present study there were variations in the origin of the artery and differences from right to left were also noted. The most common source of origin was abdominal aorta independently ie in 35 cases, and next to it was the celiac trunk ie 3 cases as shown in fig. 1 . In two cases both the IPAs were arising from CT independently but in one of them it arose from a common trunk as shown in fig.2. and one more also revealed an accessory RIPA which was arising in common with accessory renal artery and middle suprarenal artery on the same side from the Right renal artery as shown in fig.3. In one cadaver multiple variations were found on the left side. All upper lateral branches of abdominal aorta ie Inferior phrenic artery, Middle supra renal artery \& Renal artery arose from a common trunk with inferior suprarenal artery arising from the left renal artery (1 case) as shown in fig. 4 and on the left side Inferior phrenic artery alone arose from celiac trunk. In one more case Inferior phrenic artery , 2 Middle supra renal \& 2 Iinferior suprarenal arteries arose from a common trunk from a point where renal artery originated from the abdominal aorta (fig.5).

\section{Discussion}

In view of insufficient information regarding the anatomical variations of IPA and to add on to the existing anatomical literature, present study was considered, which revealed good number of variations which would be of significant value during retro peritoneal surgeries. Embryologically Celiac trunk and IPA are derived from six pairs of ventral splanchnic branches. During fetal development, these branches span and disappear, however the persistence of longitudinal channels between these primitive vessels may lead to vascular variations. IPA is said to be formed by the persistent superior artery of irregular series of arterial vessels called Rete arteriosus Urogenitale1,6, In a study by Thejodhar Pulakunta, the origin of the IP arteries from the Celiac trunk was observed in two cases $(6.25 \%)$, one from the left gastric artery $(3.125 \%)$ and one from the right renal artery $(3.125 \%)$ out of the 32 cadavers. In the remaining 28 cases it had its normal origin from the abdominal aorta.7 A study by Dong Il Gwon involving 383 interventional procedures related to the IPA revealed that right and left IPAs originate with almost equal frequency from the aorta and celiac axis and with lesser frequency from the renal arteries 8. Various other sites of origin-such as the left gastric, hepatic, superior mesenteric, spermatic, and adrenal arteries-were also seen. In instances where IPA is arising from renal artery or in common with renal artery, the suprarenal arteries are rarely derived from other than the renal arteries as they contribute mainly to the arterial supply of the adrenal glands and are thus important in angiographic examinations for adrenal lesions. 9 This has to be kept in mind in clamping the renal pedicle in Nephrectomy, when the entire blood supply of suprarenal gland on that side can be compromised by ligating the renal artery proximal to the origin of IPA.5 Modern surgical and radiological techniques require normal as well as variants in anatomy. The IPA which is a major extra hepatic source of hepato cellular carcinoma is useful in evaluating the efficacy and safety of (TOCE ) Tans catheter Oily Chemo embolisation Technique via IPA.5, 6. Knowledge of the variations of IPA can significantly assist in the management of Liver transplants, Biliary tract surgery and Liver tumour treatment. The left IPA may serve as major source of blood supply when both right and left gastric arteries get occluded.7 Knowledge of such variations show that surgeons must be cautious to avoid unintentional sectioning of small-caliber arteries, as it may occur during celiac artery decompression in compression syndrome of the celiac trunk by the median arcuate ligament.8, 9 .

\section{Conclusion}

A thorough knowledge of the vascular anatomy and variations of the IPA is critical to effective interventional treatment of the pathologic conditions related to the IPA which has to be supplemented with Careful interpretation of the angiographic findings of the same if needed.

\section{References}

[1]. Gray's Anatomy.Susan Standring et al.40 ${ }^{\text {th }}$ ed.Elsivier.2008;p.1198.

[2]. Ronald A Bergman.et ai.Illustrated encyclopedia of anatomical variations:Opus11:Cardio vascular system:Arteries:Abdomen:p.76

[3]. Soni s Wadhwa A. Multiple variations in the paired arteries of Abdominal Aorta - Clinical Implications JCDR 2010 June ;(4):262225

[4]. Origin of a common trunk for the Inferior phrenic arteries from the right renal artery:a new anatomic vascular variant with clinical implications.On Topaz Allyne Topaz Pritam R et al Cardiovascular Revascularization Medicine.11. 2010, 57-62.

[5]. Multiple aberrant coeliac trunk ramifications .Paraskevas G K ,Raikos .Singapore Med J 2011;52(7):e 147.

[6]. Felix. The development of urogenital organs. Mannual of human embryology.V.II .London.

[7]. Thejodhara Pulakunta, Bhagath Kumar Potu, Vasavi Rakesh Gorantla et al .J Vas Bras 2007 sept : 6(3)

[8]. Inferior Phrenic Artery: Anatomy, Variations, Pathologic Conditions, and Interventional ManagementDong Il Gwon, MD, GiYoung Ko, MD, Hyun-Ki Yoon, MD, Kyu-Bo Sung, MD, May 2007 RadioGraphics, 27, 687-705

[9]. Inferior phrenic arteries: angiographic anatomy, variations, and catheterization techniques for transcatheter arterial chemoembolization . Miyayama, Shiro; Yamashiro, Masashi; Yoshie, Yuichi. Japanese Journal of Radiology, Volume 28, Number 7 


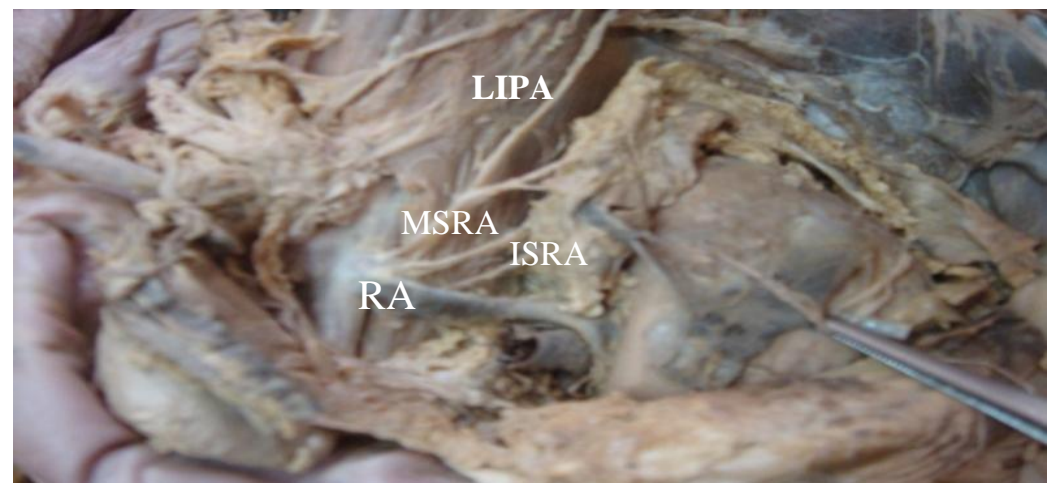

Fig.4 Inferior phrenic artery(IPA), Middle supra renal artery (MSRA) \& Renal artery (RA) arising from a common trunk

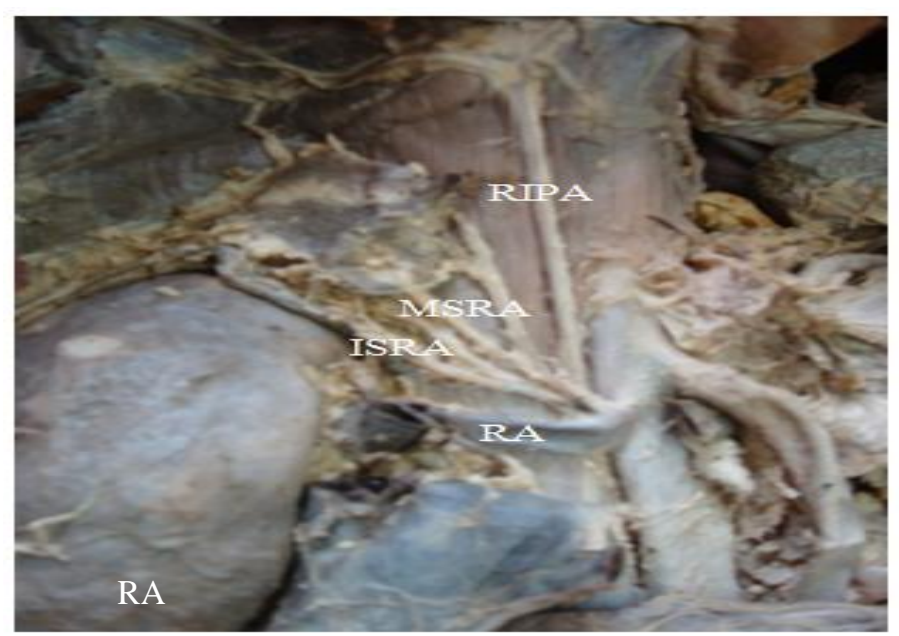

Fig.5. Inferior phrenic artery (IPA), 2 Middle supra renal (MSRA) \& 2 Inferior suprarenal arteries (ISRA)arising in common from a point where renal artery(RA) originated from the abdominal aorta.

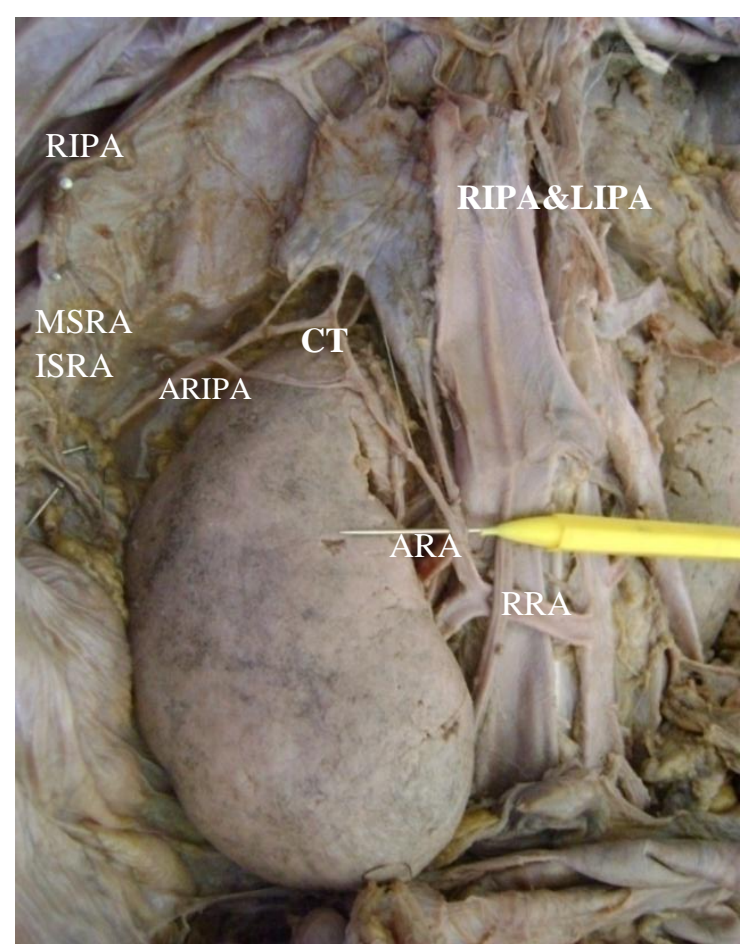

Fig.3. IPAs arising from Celiac trunk (CT) with accessory RIPA arising in common with accessory renal artery(ARA) and middle suprarenal artery from the Right renal artery(RRA) 